\title{
Generation of customized orbital implant templates using 3-dimensional printing for orbital wall reconstruction
}

\author{
Sunah Kang ${ }^{1} \cdot$ Jaeyoung Kwon ${ }^{2,3} \cdot$ Chan Joo Ahn ${ }^{1} \cdot$ Bita Esmaeli $^{4} \cdot$ Guk Bae Kim ${ }^{2,3} \cdot$ Namkug Kim $\mathbb{D}^{2,3} \cdot$ Ho-Seok Sa,4
}

Received: 3 May 2018 / Revised: 19 June 2018 / Accepted: 11 July 2018 / Published online: 28 August 2018

(c) The Royal College of Ophthalmologists 2018

\begin{abstract}
Objectives To describe and evaluate a novel surgical approach to orbital wall reconstruction that uses three-dimensionally (3D) printed templates to mold a customized orbital implant.

Methods A review was conducted of 11 consecutive patients who underwent orbital wall reconstruction using 3D-printed customized orbital implant templates. In these procedures, the orbital implant was 3D pressed during surgery and inserted into the fracture site. The outcomes of this approach were analyzed quantitatively by measuring the orbital tissue volumes within the bony orbit using computed tomography.

Results All 11 orbital wall reconstructions (6 orbital floor and 5 medial wall fractures) were successful with no post operative ophthalmic complications. Statistically significant differences were found between the preoperative and post operative orbital tissue volumes for the affected orbit $\left(24.00 \pm 1.74\right.$ vs $\left.22.31 \pm 1.90 \mathrm{~cm}^{3} ; P=0.003\right)$. There was no statistically significant difference found between the tissue volume of the contralateral unaffected orbit and the affected orbit after reconstruction $\left(22.01 \pm 1.60 \mathrm{~cm}^{3}\right.$ vs $\left.22.31 \pm 1.90 \mathrm{~cm}^{3} ; P=0.182\right)$.

Conclusion 3D-printed customized orbital implant templates can be used to press and trim conventional implantable materials with patient-specific contours and sizes for optimal orbital wall reconstruction. It is difficult to design an orbital implant that exactly matches the shape and surface of a blowout fracture site due to the unique 3D structure of the orbit. The traditional surgical method is to visually inspect the fracture site and use eye measurements to cut a two-dimensional orbital implant that corresponds to the anatomical structure of the fracture site. However, implants that do not fit the anatomical structure of a fracture site well can cause complications such as enophthalmos, diplopia and displacement of the implant.
\end{abstract}

\section{Introduction}

Three-dimensionally (3D) printing technology has become widely available for clinical use and allows surgeons to perform far more accurate orbital reconstructions [1-6].

$\triangle$ Ho-Seok Sa

lineblue@hanmail.net

1 Department of Ophthalmology, Asan Medical Center, University of Ulsan College of Medicine, Seoul, Korea

2 Department of Radiology, Asan Medical Center, University of Ulsan College of Medicine, Seoul, Korea

3 Department of Convergence Medicine, Asan Medical Center, University of Ulsan College of Medicine, Seoul, Korea

4 Ophthalmology Section, Department of Plastic Surgery, The University of Texas M. D. Anderson Cancer Center, Houston, TX, USA
Although there is currently a cost limitation with the use of 3D printers to generate biocompatible materials (i.e., titanium or porous polyethylene), and it is not yet possible to 3D print porous polyethylene with embedded titanium implants, the recent development and application of 3D printing techniques to orbital wall reconstruction has enabled surgeons to design implants that closely fit the shape and surface of an individual fracture site. In addition, operation times can be reduced using customized implants [5, 6]. 3Dprinted custom-made orbital implants can be particularly useful for surgeon with less experience who may not be as facile at empirically designing the orbital implants.

In our present study, we made customized 3D-printed orbital implant templates and used them intraoperatively in patients who had sustained orbital wall fractures, and we evaluated the effect of orbital reconstruction quantitatively by measurement of orbital tissue volume within the bony orbit before and after surgery and also between both orbits in each patient. 


\section{Materials and methods}

\section{Patients}

A retrospective review was conducted in all consecutive patients who had a traumatic "blowout" fracture and underwent an orbital wall reconstruction using 3D-printed customized orbital implant templates by a single surgeon (H.-S.S.) at Asan Medical Center, Seoul, Korea, between November 2016 and July 2017. Patients with other combined facial bone fractures, bilateral blowout fractures or accompanying orbital disease affecting the orbital tissue volume, such as orbital mass and thyroid eye disease, were excluded. Each of the included patients underwent a baseline ophthalmic examination prior to surgery that included the following assessments: visual acuity, a slit lamp examination, a Goldmann diplopia test by perimetry, a Hess screen test to check extra-ocular muscle motility, and Hertel exophthalmometry to measure enophthalmos. Preoperative and post operative facial bone computed tomography (CT) scans were conducted in all of the study patients.

\section{Preparation of customized orbital implant templates}

We developed a new technique for making customized orbital implant templates that can be used intraoperatively. The patient's orbit including the fracture site was analyzed using Mimics and 3-Matic software (Materialise, Leuven, Belgium) to create a virtual 3D orbital implant model for the fracture site (Fig. 1a). Using the surface construction tool in the software, the fracture site was reconstructed to simulate the natural contour of the orbit. A virtual 3D orbital implant model was then created to fit the shape and surface of the fracture site and converted into a stereolithography (STL) file. The templates and press were printed using a Projet 3510SD device (3DSystems, Inc., Rock Hill, SC) with $32 \mu \mathrm{M}$ resolution (Fig. 1b). We used UV curable plastic with a wax supporter which costs around $\$ 640$ and $\$ 380$ per $\mathrm{kg}$, respectively, with an estimated cost of $\$ 100$ for the templates (upper and lower body) and $\$ 150$ for a press. After melting of the wax supporter, the implant was cleaned with isopropanol and sterilized with ethylene oxide gas at $55^{\circ} \mathrm{C}$ for intraoperative use.

\section{Surgical procedure}

Orbital floor fractures were exposed through a preseptal transconjunctival incision and medial orbital wall fractures through a transcaruncular incision. After any herniated orbital contents were repositioned, orbital wall defects were reconstructed using a customized orbital implant. The customized orbital implant template was traced onto a conventional 2-dimensional implant comprising porous polyethylene with embedded titanium (SYNPOR ${ }^{\circledR}$ Implant Titanium Reinforced Fan Plates; DePuy Synthes, Inc, West Chester, PA) with all unnecessary portions removed (Fig. 1c). The implant was then placed between the upper and lower body of the templates and then inserted into a press to maintain the axis while pressing and thereby stencil the customized 3-demensional contour onto the implant (Fig. 1d). After insertion of the implant to cover the orbital wall defect, a forced duction test was performed to ensure the absence of restriction. The conjunctival wound was closed with interrupted 6-0 vicryl sutures.

\section{Orbital tissue volume measurement}

The measurements of orbital tissue volume within the bony orbit were determined from preoperative and post operative CT scans using 3-Matic software (Materialise, Leuven, Belgium) (Fig. 2). The three landmark points on the anterior reference plane were the supraorbital notch, zygomaticofrontal suture and inferior end of the anterior lacrimal crest margin. Statistics were performed with SPSS software version 21.0 (IBM, Armonk, NY) using the Wilcoxon signed rank test. Differences were considered statistically significant at $P<0.05$.

\section{Results}

Eleven patients ( 7 men and 4 women) with a median age of 33 years (range: $20-70$ years) were included in the analysis. The median follow-up period was 17 weeks (range: 2-42 weeks). Table 1 summarizes the patients' clinical findings at baseline and postoperatively. Six patients had orbital floor fracture and five patients had a medial wall fracture (Fig. 3). All patients underwent primary surgical treatment for a blowout fracture. Indications for surgery included persistent diplopia in four patients, significant enophthalmos (>2 mm) at baseline in two patients, and large fracture involving over $50 \%$ of wall in five patients. These symptoms and signs were resolved in all patients after the surgery. None of the 11 study patients experienced post operative ophthalmic complications such as wound infection, dehiscence, hematoma, foreign body reaction, displacement of implant, or visual acuity disturbance.

The tissue volume within the bony orbit of the affected orbit, which was increased by trauma-induced herniation, was significantly decreased after the surgical reconstruction $\left(24.00 \pm 1.74\right.$ vs $22.31 \pm 1.90 \mathrm{~cm}^{3} ; P=0.03$; Table 2$)$. There was no statistically significant difference found between the tissue volume within the bony orbit of the affected orbit after reconstruction and the contralateral unaffected orbit $(22.31$ \pm 1.90 vs $22.01 \pm 1.60 \mathrm{~cm}^{3} ; P=0.182$ ). 
Fig. 1 Technique for preparation of a customized orbital implant template. a Preoperative virtual 3D orbital implant model for the fracture site. b Templates and press. The design of the upper and lower body of the templates was based on the virtual 3D orbital implant shown in a and c. Tracing of a template onto porous polyethylene with an embedded titanium implant and cutting out of the unnecessary parts. d After placement of the porous polyethylene with embedded titanium implant between the upper and lower body of the templates, it was inserted into the press to maintain the axis while pressing
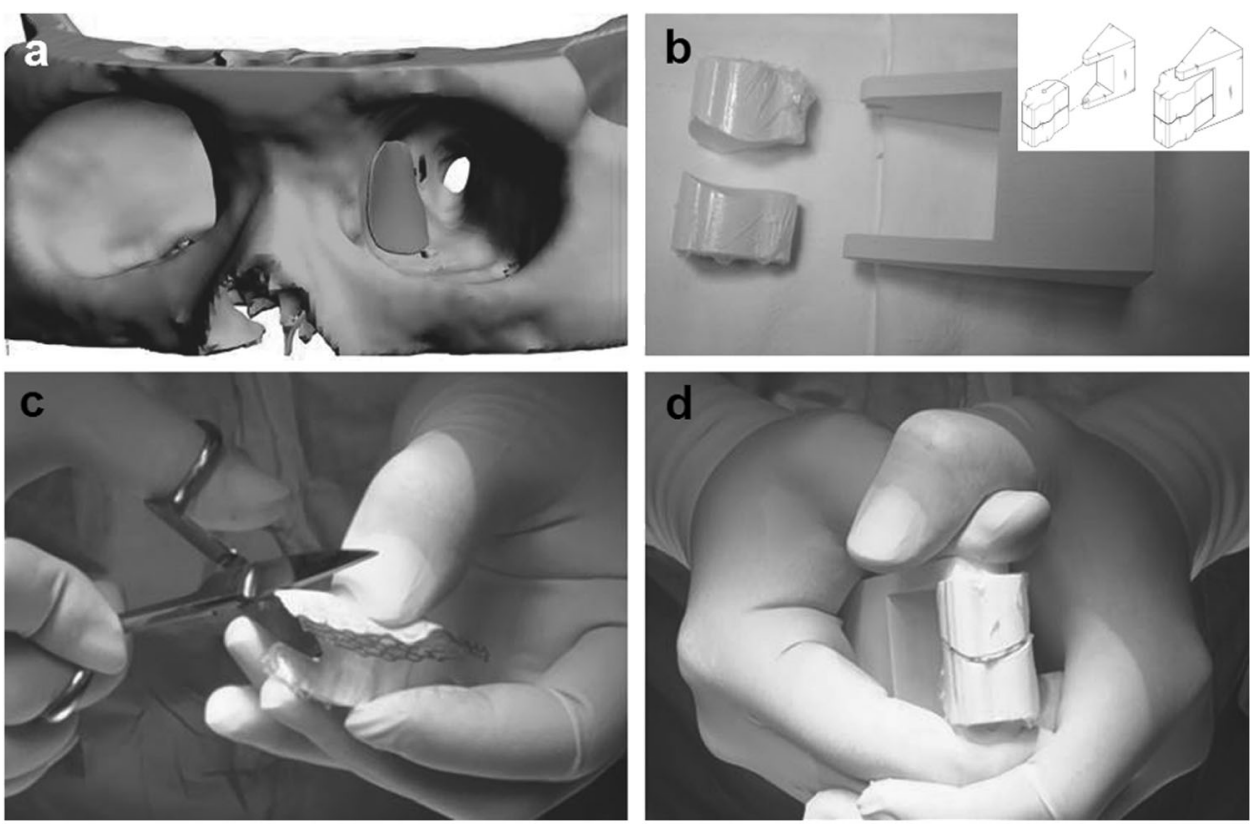

a

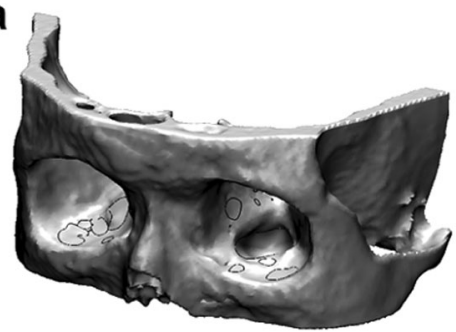

b

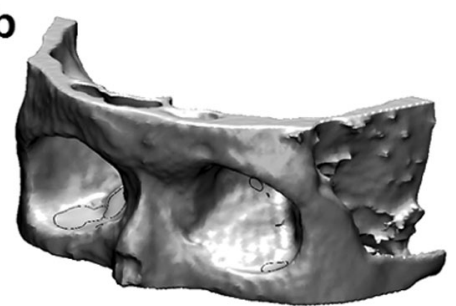

Fig. 2 Three-dimensional volumetric measurements of the orbital tissue within the bony orbit. a Preoperative assessment. The arrow indicates the herniated orbital tissue through the bony defect of the left orbital floor fracture. b Post operative assessment. The herniated orbital tissue was reduced

\section{Discussion}

Our findings suggest that 3D-printed customized orbital implant templates can be used intraoperatively for primary reconstruction of commonly encountered blowout fractures of the orbital walls. We confirmed that this method of orbital wall reconstruction has an excellent outcome through quantitative, rather than qualitative, evaluation of orbital volume before and after surgery.

Facial bone reconstruction using 3D printing has evolved over time. Since the orbital wall is very thin with sensitive nearby structures such as the eyeball, optic nerve, and extraocular muscles, the development of 3D technology for the orbital wall has been slower than that for other facial bones. The earliest described technique in this regard involved application of an orbital implant by direct molding onto a 3D-printed skull model as the surgical guide [7, 8]. More recently, population-based pre-bent implants have been reported in several studies [9-13]. These implants have shown better outcomes than those achieved using a conventional plane 2D implants. However, considering that individual patients have different orbital morphologies, these earlier method were limited by being unable to take full advantage of 3D printing technology to produce fully customized implants. Also, there is technology available for directly printing a suitable biocompatible material for implantation into the orbit, although this is largely limited to titanium [4]. Pure titanium implants are usually far from being ideal for orbital wall reconstruction for the most commonly occurring simple one-wall blowout fractures and are associated with complications of orbital adherence syndrome [14]. Notably also, direct printing is currently very expensive and requires specific equipment.

The method we presented in our report has several advantages over existing technologies. First, our approach makes it possible to generate a size and contour (configuration) that precisely fits the fracture site in a fully customized manner and that is different therefore from population-based pre-bent implants. Second, our method is not very technically challenging. A notable aspect is that it differs from mirroring as it does not refer to the shape of the contralateral unaffected orbit and can directly restore the fracture site in the affected orbit. If the boundaries of the 


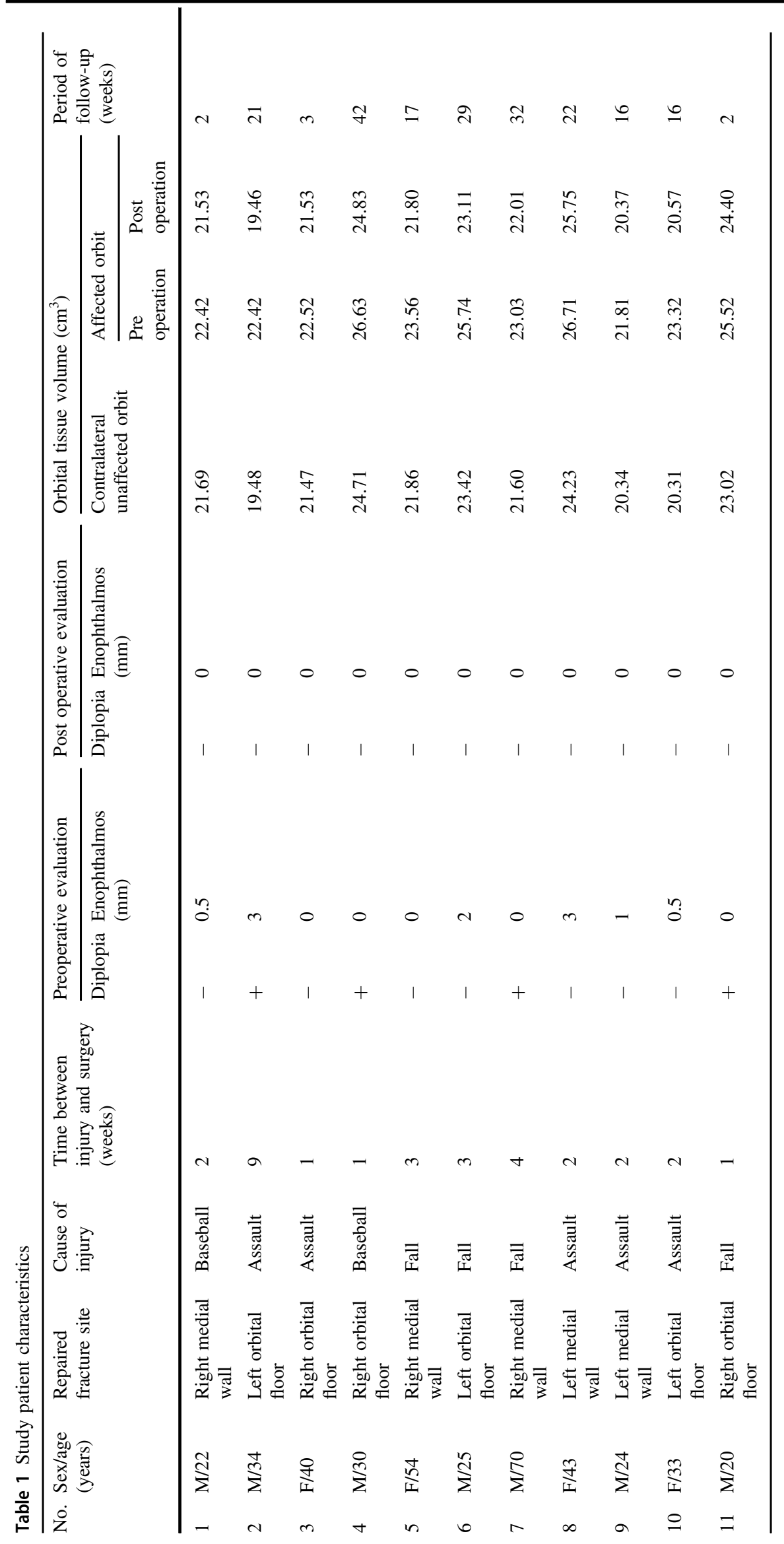



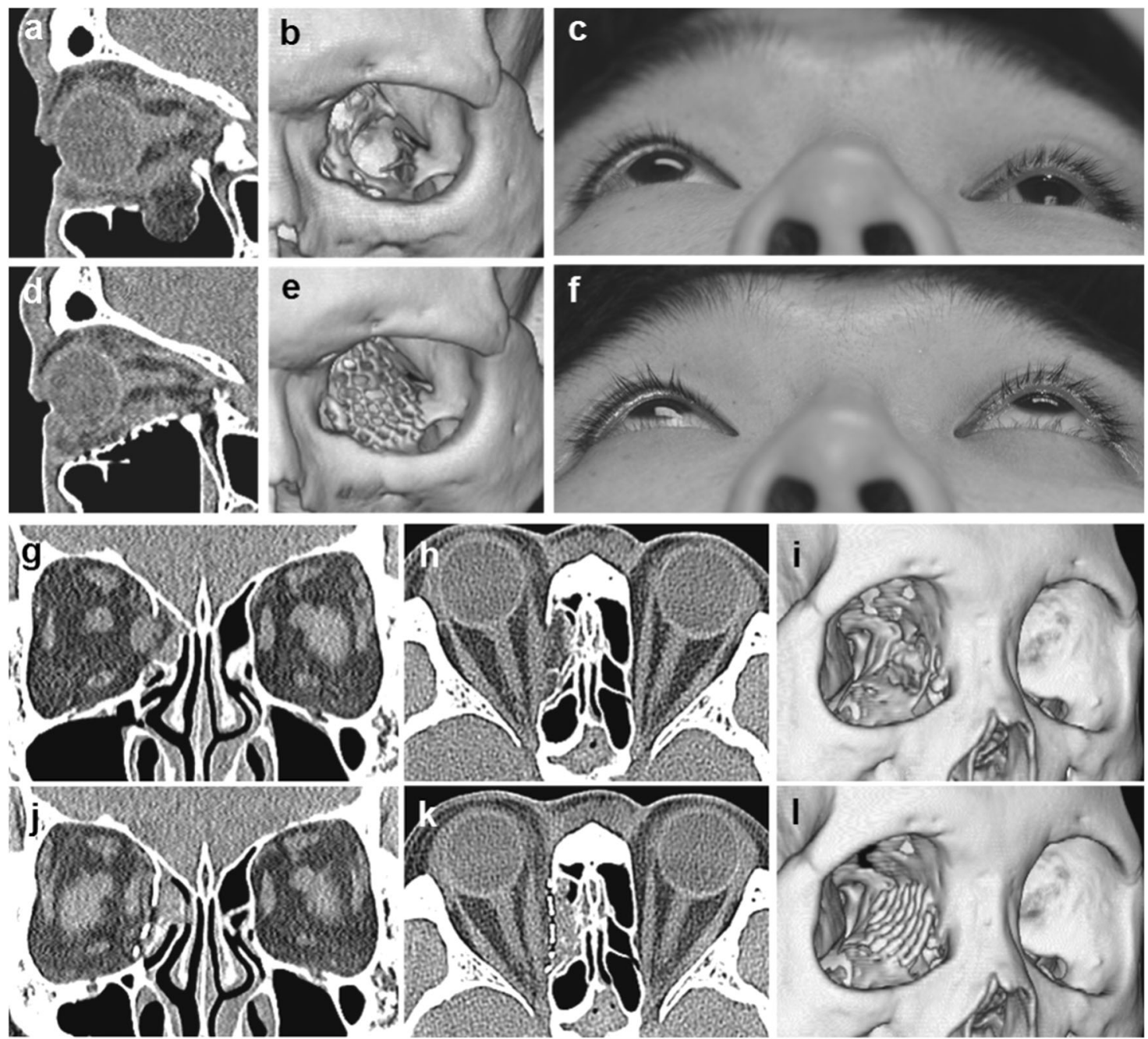

Fig. 3 Preoperative $(\mathbf{a}, \mathbf{b}, \mathbf{c})$ and post operative $(\mathbf{d}, \mathbf{e}, \mathbf{f}) \mathrm{CT}$ scans and photo of patient 2. The left orbital floor fracture in sagittal view (a), three-dimensional reconstruction (b), and enophthalmos in left eye (c) are shown. Post operative images $(\mathbf{d}, \mathbf{e})$ revealed a good size and contour of the implant pressed and trimmed using a customized orbital implant template generated by 3-dimensional printing. The

Table 2 Three-dimensional volumetric measurements of the contralateral unaffected and affected orbits in the study population

Mean orbital tissue volume within the bony $P$-value orbit $\left(\mathrm{cm}^{3}\right)$

\begin{tabular}{lll}
\hline $\begin{array}{l}\text { Contralateral unaffected } \\
\text { orbit } \\
(n=11)\end{array}$ & $\begin{array}{l}\text { Affected orbit } \\
(n=11)\end{array}$ & \\
\hline 22.06 & 24.00 & 0.003 \\
22.01 & 22.31 & 0.182 \\
0.859 & 0.003 & \\
\hline
\end{tabular}

fracture are drawn manually, the template can be modeled semi-automatically using a surface construction tool in a commercial software program that computes the curvature and fills the surface. Third, surgeons can still select their enophthalmos in left eye was improved (f). Preoperative $(\mathbf{g}, \mathbf{h}, \mathbf{i})$ and post operative $(\mathbf{j}, \mathbf{k}, \mathbf{l})$ CT scans of patient 5 . The right medial wall fracture in coronal view (g), axial view (h), and three-dimensional reconstruction (i) are shown. Post operative images $(\mathbf{j}, \mathbf{k}, \mathbf{l})$ demonstrated optimal positioning and configuration of the customized implant

preferred commercial implants when using our approach. In addition, there is no need to use facilities for direct printing of the biocompatible material. Our technology can therefore be easily adopted by any clinic with a 3D printer and an appropriate software program.

A fourth advantage of our technique is cost reduction. Direct 3D printing of titanium implants costs between $\$ 3700$ and $\$ 6200$ [1], and a previous study by Fan et al. [6], who also introduced an orbital implant template, reported that it costs about $\$ 310$, which had been the lowest cost reported thus far. The cost of the templates used in our patients was approximately $\$ 100$, and the press is reusable. This confirms that our method is inexpensive but still produces very satisfactory outcomes. A fifth benefit of our present approach was that the template and the press were 
used together to maximize the molding effect. Previously, Callahan et al. [4] and Fan et al. [6] have also reported the use of orbital implant templates in orbital reconstructions. In both of these prior studies, the surgeon manually shaped and trimmed the implant on the templates. We have improved the molding effect by producing the upper and lower template body separately. After placing the porous polyethylene with embedded titanium implant between the upper and lower body of templates, it is inserted into the press which maintains the axis while pressing (Fig. 1b).

Based on the surgeon's preference, various types of implants can be used with the 3D-printed customized template. We prefer porous polyethylene with embedded titanium implants because it has the advantageous properties of both porous polyethylene and titanium. Titanium mesh has significant tensile strength and can be easily contoured to fit the shape and surface of the fracture site because of its malleability and memory [15], which are essential properties for 3D molding. However, the sharp and irregular edges of titanium mesh are its disadvantages and make it difficult to insert through small incisions. With its barrier coating, a porous polyethylene with embedded titanium implant can be easily inserted through a small transconjunctival incision and serve as a smooth nonadherent barrier that also maintains its malleability and memory.

We further analyzed the effects of orbital wall reconstruction quantitatively by measuring orbital tissue volumes within the bony orbit with a computer-aided design program. During the early post-traumatic period, enophthalmos may not be observed or measurable because of orbital soft tissue swelling [16]. Hence, orbital tissue volume measurements including the herniated area are useful when determining the effect of an orbital wall reconstruction [16]. We observed a significant reduction in the orbital tissue volume within the bony orbit after orbital wall reconstruction surgery in our current patient cohort. Such orbital tissue volume measurement techniques can also be applied to other areas such as orbital prosthesis and enophthalmos correction.

There were some notable limitations of our present study including the small number of patients and the relatively short follow-up interval for some of our cases. However, Wi et al. [16] have previously reported no significant change in the orbital volume measured at two time points after orbital wall reconstruction, i.e., immediately after surgery and at a final follow-up at a minimum of 6 months. The post operative orbital tissue volume within the bony orbit is thus unlikely to change in any significant way, regardless of the follow-up interval. An additional limitation of this present study was that we excluded patients with other accompanying facial bone fractures or with a combined orbital floor and medial wall fracture involving the inferomedial strut. The extent of complex orbital wall fracture to which our technique can be applied is therefore not fully unclear. Future studies should attempt this technique and evaluate its utility for more extensive and complex orbital fractures.

\section{Summary}

\section{What was known before}

- The traditional orbital wall reconstruction of blowout fracture is to visually inspect the fracture site and use eye measurements to cut a two-dimensional orbital implant that corresponds to the anatomical structure of the fracture site.

- The implants that do not fit the anatomical structure of a fracture site well can cause complications such as enophthalmos, diplopia, and displacement of the implant.

\section{What this study adds}

- We introduced the surgical technique of orbital wall reconstruction using 3D-printed customized orbital implant templates with low cost and quantitatively demonstrated optimal reconstruction of anatomic contours.

Funding This study was supported by a grant (Grant Number 2016-7020) from the Asan Institute for Life Sciences, Asan Medical Center, Seoul, Korea.

\section{Compliance with ethical standards}

Conflict of interest The authors declare that they have no conflict of interest.

\section{References}

1. Vehmeijer M, van Eijnatten M, Liberton N, Wolff J. A novel method of orbital floor reconstruction using virtual planning, 3dimensional printing, and autologous bone. J Oral Maxillofac Surg. 2016;74:1608-12.

2. Park SW, Choi JW, Koh KS, Oh TS. Mirror-imaged rapid prototype skull model and pre-molded synthetic scaffold to achieve optimal orbital cavity reconstruction. J Oral Maxillofac Surg. 2015;73:1540-53.

3. Oh TS, Jeong WS, Chang TJ, Koh KS, Choi JW. Customized orbital wall reconstruction using three-dimensionally printed rapid prototype model in patients with orbital wall fracture. J Craniofac Surg. 2016;27:2020-4.

4. Callahan AB, Campbell AA, Petris C, Kazim M. Low-cost 3D printing orbital implant templates in secondary orbital reconstructions. Ophthal Plast Reconstr Surg. 2017;33:376-80.

5. Zielinski R, Malinska M, Kozakiewicz M. Classical versus custom orbital wall reconstruction: Selected factors regarding surgery and hospitalization. J Craniomaxillofac Surg. 2017;45:710-5. 
6. Fan B, Chen H, Sun YJ, Wang BF, Che L, Liu SY, et al. Clinical effects of 3-D printing-assisted personalized reconstructive surgery for blowout orbital fractures. Graefes Arch Clin Exp Ophthalmol. 2017;255:2051-7.

7. Metzger MC, Schon R, Schulze D, Carvalho C, Gutwald R, Schmelzeisen R. Individual preformed titanium meshes for orbital fractures. Oral Surg Oral Med Oral Pathol Oral Radiol Endod. 2006;102:442-7.

8. Mustafa SF, Evans PL, Bocca A, Patton DW, Sugar AW, Baxter PW. Customized titanium reconstruction of post-traumatic orbital wall defects: a review of 22 cases. Int J Oral Maxillofac Surg. 2011;40:1357-62.

9. Metzger MC, Schon R, Weyer N, Rafii A, Gellrich NC, Schmelzeisen R, et al. Anatomical 3-dimensional pre-bent titanium implant for orbital floor fractures. Ophthalmology. 2006;113:1863-8.

10. Scolozzi P, Momjian A, Heuberger J, Andersen E, Broome M, Terzic A, et al. Accuracy and predictability in use of AO threedimensionally preformed titanium mesh plates for posttraumatic orbital reconstruction: a pilot study. J Craniofac Surg. 2009;20:1108-13.
11. Lee KM, Park JU, Kwon ST, Kim SW, Jeong EC. Threedimensional pre-bent titanium implant for concomitant orbital floor and medial wall fractures in an East asian population. Arch Plast Surg. 2014;41:480-5.

12. Huempfner-Hierl H, Doerfler HM, Kruber D, Hierl T. Morphologic comparison of preformed orbital meshes. J Oral Maxillofac Surg. 2015;73:1119-23.

13. Gordon CR, Susarla SM, Yaremchuk MJ. Quantitative assessment of medial orbit fracture repair using computer-designed anatomical plates. Plast Reconstr Surg. 2012;130:698e-705e.

14. Lee HB, Nunery WR. Orbital adherence syndrome secondary to titanium implant material. Ophthal Plast Reconstr Surg. 2009;25:33-36.

15. Garibaldi DC, Iliff NT, Grant MP, Merbs SL. Use of porous polyethylene with embedded titanium in orbital reconstruction: a review of 106 patients. Ophthal Plast Reconstr Surg. 2007;23:439-44.

16. Wi JM, Sung KH, Chi M. 'Orbital volume restoration rate after orbital fracture'; a CT-based orbital volume measurement for evaluation of orbital wall reconstructive effect. Eye. 2017;31:713-9. 